\title{
Resonant enhancement of relativistic electron fluxes during geomagnetically active periods
}

\author{
I. Roth ${ }^{1}$, M. Temerin ${ }^{1}$, M. K. Hudson ${ }^{2}$ \\ ${ }^{1}$ Space Sciences Laboratory, University of California, Berkeley, CA 94720, USA \\ e-mail: ilan@ssl.berkeley.edu \\ ${ }^{2}$ Physics and Astronomy Department, Dartmouth College, Hanover, NH 03755, USA
}

Received: 30 June 1998 / Revised: 26 October 1998 / Accepted: 27 October 1998

\begin{abstract}
The strong increase in the flux of relativistic electrons during the recovery phase of magnetic storms and during other active periods is investigated with the help of Hamiltonian formalism and simulations of test electrons which interact with whistler waves. The intensity of the whistler waves is enhanced significantly due to injection of $10-100 \mathrm{keV}$ electrons during the substorm. Electrons which drift in the gradient and curvature of the magnetic field generate the rising tones of VLF whistler chorus. The seed population of relativistic electrons which bounce along the inhomogeneous magnetic field, interacts resonantly with the whistler waves. Whistler wave propagating obliquely to the magnetic field can interact with energetic electrons through Landau, cyclotron, and higher harmonic resonant interactions when the Doppler-shifted wave frequency equals any (positive or negative) integer multiple of the local relativistic gyrofrequency. Because the gyroradius of a relativistic electron may be the order of or greater than the perpendicular wavelength, numerous cyclotron, harmonics can contribute to the resonant interaction which breaks down the adiabatic invariant. A similar process diffuses the pitch angle leading to electron precipitation. The irreversible changes in the adiabatic invariant depend on the relative phase between the wave and the electron, and successive resonant interactions result in electrons undergoing a random walk in energy and pitch angle. This resonant process may contribute to the 10-100 fold increase of the relativistic electron flux in the outer radiation belt, and constitute an interesting relation between substorm-generated waves and enhancements in fluxes of relativistic electrons during geomagnetic storms and other active periods.
\end{abstract}

Key words. Magnetospheric physics (energetic particles, trapped; plasma waves and instabilities; storms and substorms).

Correspondence to: I. Roth

\section{Introduction}

Magnetic storms cause some of the largest geomagnetic field deformations. The source of these strong perturbations originates at the Sun and they are believed to be triggered by a persistent southward interplanetary magnetic field. Generally, a magnetic storm is characterised by an enhancement in the ring current, due to the injection of ions by strong convective electric field. An additional important storm characteristic is the behaviour of energetic electrons during different phases of the storm. The evolution of relativistic electrons in the outer radiation belt depends on the time scale of magnetic perturbations and on the different characteristic frequencies of electron dynamics. If the perturbation time scale is much longer than that of the quasiperiodic motion of a particle in geomagnetic field, the corresponding adiabatic invariant is conserved. Conservation of the three invariants during the main phase of the storm together with Liouville's theorem requires that the enhanced ring current, which decreases the inner magnetospheric magnetic field, causes a decrease in the electron flux as the electrons move to higher $L$ shells. The resulting decrease in relativistic electron fluxes at this phase has been observed by numerous satellites like SAMPEX, WIND and POLAR (Baker et al., 1997; Reeves et al., 1998). At the recovery phase one observes an increase up to two orders of magnitude in the flux of relativistic electrons ( $\mathrm{Li}$ et al., 1998; Reeves et al., 1998). During active times the flux increase of very energetic electrons $(>3 \mathrm{MeV})$ at lower $L$ shells often precede the increase of electrons with the same first and second adiabatic invariant at higher $L$ and results in a peak of the distribution $f(L)$ around $L=4.5$ (Selesnick and Blake, 1997). The decay phase is characterised partly by adiabatic behaviour, as well as by radial diffusion (which violates the third invariant) and by pitch angle scattering (which violates the first two adiabatic invariants) (e.g., Mcllwain 1996; Li et al., 1998). The increase in the relativistic electron flux during active times and in the storm recovery phase is 
the least understood process and is addressed in the present investigation.

Large flux enhancements above quiet-time levels at $L \sim 4-5$ have been observed during geomagnetic storms, as well as during active periods following the arrival of high speed solar wind streams at 1 AU. GPS data show increase in the flux of $>1 \mathrm{MeV}$ electrons at $L=4.5$ during the January 1997 coronal mass ejection (CME) January 1997 event, without significant flux increase initially at higher $L$ values. The HIST instrument on the POLAR satellite (Blake et al., 1996) measured numerous large enhancements in $f(L)$ at low $L$ shells during active periods, including the January CME event (Selesnick and Blake 1997, 1998). In other active periods the enhanced fluxes of energetic electrons (0.7-3.0 MeV) at $L \sim 4-5$ probably are not a result of diffusion from higher $L$ shells (Selesnick and Blake, 1998), which determines the spatial distribution during quiet periods. Additionally, an analysis of the November 3-4, 1993 high-speed solar wind stream event showed that the phase space density in the solar wind is insufficient to explain the increase of relativistic outer radiation belt electrons (Li et al., 1997). Therefore, enhancement in the high energy electron fluxes at lower L-Shells (Paulikas and Blake, 1979) indicates a possible existence of additional processes which operate at this region where a local heating mechanism may be operative for relativistic electrons.

\section{Energization mechanisms}

Enhancements in energetic radiation belt electron fluxes at invariant shells $L \sim 4-5$ may be the result of several processes; (a) direct injection by a strong electromagnetic pulse which abruptly deforms the magnetic configuration and energises electrons and protons by breaking their third invariant when a subset of particles is in a phase with a single "coherent" wave ( $\mathrm{Li}$ et al., 1993) or when they are subjected to a large-amplitude ULF waves (Hudson et al., in press, 1999); (b) radial diffusion of a distribution function with a positive gradient in $L$ which violates the flux invariant by a random walk due to broad-band, small-amplitude, lowfrequency electromagnetic perturbations (Schults and Lanserotti, 1974; Selesnick et al., 1997); (c) resonant interaction with higher frequency waves on the order of gyration or bounce time scales, with violation of the first two invariants. Mechanism (a) occurs infrequently and requires a large sudden commencement pulse excited by a fast interplanetary shock wave or intense ULF waves excited by a strong coronal mass ejection perturbation. Mechanism (b) tends to flatten the distribution $f(L)$ and cannot describe separately the increase in the lower $L$ shells and the formation of maxima at these $L$ values. Mechanism (c) requires recurrent increase in the power of waves which interact with a seed population of energetic electrons and diffuse them in energy and pitch angle. This mechanism involves resonant interaction with electrons bouncing and gyrating along the inhomogeneous dipole magnetic field, $\omega-k_{\|} v_{\|}-n \Omega / \gamma \sim 0$, where the wave is characterized by its frequency $\omega$ and parallel wave number $k_{\|}$and the resonating electron by its parallel velocity $v_{\|}$, local gyrofrequency $\Omega$ and the relativistic factor $\gamma$, while the integer $n$ denotes the harmonic of the cyclotron interaction. In the next sections we focus on the interaction of these waves with relativistic electrons.

\section{Flux enhancement scenario}

A possible mechanism for energization of the relativistic electrons in the outer radiation belt is due to interaction with whistler waves. Whistler waves play an important role in the theory of trapped magnetospheric electrons but they are usually invoked as a loss mechanism. Resonant interaction with whistler waves, which occurs when the cyclotron resonance condition is satisfied, is considered as viable mechanism for the formation of the slot region in the inner magnetosphere by electron precipitation (Lyons and Thorne, 1973). Pitch angle scattering of electrons into the loss cone by whistler waves leads to a decay of the outer electron belt during quiet periods. These waves were also shown to be a source of heating of $<1 \mathrm{keV}$ electrons by fundamental cyclotron $(n=-1)$ and Landau $(n=0)$ interactions (Thorne and Horne, 1994, 1996). If the wave is propagating parallel to the magnetic field only the lowest order resonant interaction, where the electron and wave are moving in opposite directions along the magnetic field, produces changes in the electron pitch angle and energy. There exists an inverse correlation between the energy change and the pitch angle change: a decrease in the pitch angle leads to an increase in the energy. For typical plasma parameters around the dense plasmasphere, the dominant effect of the interaction is pitch angle scattering and while it is acknowledged that pitch angle scattering necessarily involves some energy change, it can be shown that this change is small (e.g., Hasegawa, 1975). Thus, any large change in energy involves a larger relative change in pitch angle, which will scatter electrons into the loss cone. However, if the whistler wave is not propagating exactly parallel to the magnetic field, higher order resonant interactions can occur when the Dopplershifted wave frequency equals any (positive or negative) integer multiple of the gyrofrequency. Interaction of suprathermal $(100 \mathrm{eV})$ electrons with oblique whistler waves was used for the study of electron precipitation (Jasna et al., 1992). For relativistic electrons moving along an inhomogeneous field there exist typically several harmonic interactions corresponding to the first few positive and negative integers where the resonant condition is satisfied, and since at these energies the gyroradius of the electron may be on the order of or greater than the perpendicular wavelength, the strength of the interaction at the higher harmonics is of the same order as at the fundamental. Since now the energy and pitch angle changes are no longer correlated, the electrons undergo more or less independent random walks in energy and pitch angle (and in the adiabatic invariant). Thus, the mechanism operates mainly for 
energetic electrons whose distribution is heated even as some electrons are scattered into the loss cone.

Electromagnetic chorus whistler waves were observed over a broad range of $L$ shells between plasmapause and magnetopause. The high-latitude chorus generation mechanism may be due to loss-cone distributions around the minima of magnetic field formed due to compression of the dayside magnetosphere (Roederer, 1970; Tsurutani and Smith, 1977). At low latitudes numerous geostationary satellites have observed injections of electrons with moderate energy $(10-100 \mathrm{keV})$ during magnetic substorms (Reeves et al., 1998). Recent statistical investigations reveal that these substormrelated electrons are correlated to VLF chorus on the whistler branch (Smith et al., 1996). Electrons, which drift radially due to convection electric fields and eastward due to magnetic gradient and curvature, exhibit energy dispersion, forming distributions which may destabilise whistler waves. The faster (slower) electrons excite the lower (higher) frequencies, giving rise to the ascending tones of the chorus. This whistler chorus, which may be considered an additional signature of the substorm expansion phase, may interact with the relativistic electrons. Generally, the wave normal angle of whistler waves increases with latitude and its refraction at the plasmapuase and reflection at high latitutes allows access to regions far from its source (Church and Thorne, 1983). Similarly, it was shown that non-ducted whistler waves, which were excited by lightning discharge, occupy wide regions of the magnetosphere. Therefore the oblique whistler waves can interact efficiently with relativistic electrons during their bounces along the magnetic field. Because of the large changes in the gyrofrequency of a relativistic electron along the bounce trajectory and due to its large velocity, it can resonantly interact with multiple harmonics of the whistler wave. Wave-tracing studies (Draganov et al., 1992; Ristic-Djurovic et al., 1998) indicate that the magnetospherically reflected whistlers settle down at $L$ shell corresponding to their signal frequency, forming at a given $L$ shell a narrow-band whistler wave. In the present analysis we consider the resulting resonant interaction with monochromatic wave which violates the first and the second adiabatic invariants and results in energization of relativistic electrons on a time scale much shorter than the one due to the $L$-shell diffusion.

\section{Analysis of the resonant interaction}

Energetic electrons which bounce along the inhomogeneous magnetic field and encounter a resonance, experience irreversible changes in their adiabatic invariants. Energetic electrons with gyroradius of the order of the perpendicular wavenumber may interact with numerous harmonics of the cyclotron resonance. For a monochromatic wave the normalised relativistic Hamiltonian $H(\mathbf{x}, \mathbf{P})$ with $\mathbf{P}=m \mathbf{v} \gamma+q \mathbf{A}(\mathbf{x}) / c$ becomes

$H=\left[1+(\mathbf{p}-\mathbf{A}(\mathbf{x}))^{2}\right]^{1 / 2}+\Phi(\mathbf{x})$ where the canonical momentum $\mathbf{P}$ is normalised to $m c$, the time $t$ to the inverse equatorial gyrofrequency $\Omega_{0}^{-1}$, the spatial coordinates $x$ to $c / \Omega_{0}$, and the wave-number $\mathbf{k}=[k \sin \phi, 0, k \cos \phi]$ to $\Omega_{0} / c . \phi$ denotes the propagation angle with respect to the ambient magnetic field.

The background magnetic field is represented by the normalised potential $\mathbf{A}_{0}=x \eta(\epsilon z) \boldsymbol{y}$, with the normalised gyrofrequency $\eta(\epsilon z)=\Omega(\epsilon z) / \Omega_{0}$, where the $\epsilon$ dependence denotes the slowly changing gyrofrequency. The electric field $\left[E_{x} \cos \psi, E_{y} \sin \psi, E_{z} \cos \psi\right]$ is derived from the electrostatic $\Phi=\delta_{0} \sin \psi$ and the electromagnetic potential $\mathbf{A}=\left[\delta_{1}\left(k_{\|} / k\right) \sin \psi, \delta_{2} \cos \psi_{1}-\delta_{1}\right.$ $\left.\left(k_{\perp} / k\right) \sin \psi\right]$, with the phase $\psi=\left[\int \mathbf{k}(\epsilon z) \mathbf{x}-\omega t\right]$.

Performing the canonical transformation $F_{1}(x, \theta)=$ $0.5 \eta(\epsilon z) x^{2} \operatorname{ctg} \theta$, changing to the action-angle variables $(I, \theta)$ and expanding in $\delta_{i}$, using the Bessel decomposition, casts the Hamiltonian into $H=H_{0}+H_{1}$ where

$$
\begin{aligned}
& H_{0}\left(I, P_{\|}\right)=\left[1+2 I \eta+P_{\|}^{2}\right]^{1 / 2}=\gamma_{o} \\
& H_{1}\left(I, P_{\|}, z, \theta\right)=\sum_{n} G_{n}\left(P_{\|}, I\right) \sin \left[k_{\|} z+n \theta-\omega t\right]
\end{aligned}
$$

with

$$
\begin{aligned}
G_{n}=[ & \left.\delta_{1}\left(P_{\|} \sin \phi-n \eta \cos \phi / k_{\perp}\right) / \gamma_{o}+\delta_{o}\right] J_{n}\left[k_{\perp}(2 I / \eta)^{1 / 2}\right] \\
& +\delta_{2}\left[(2 I \eta)^{1 / 2} / \gamma_{o}\right] J_{n}^{\prime}\left[k_{\perp}(2 I / \eta)^{1 / 2}\right]
\end{aligned}
$$

The Bessel functions indicate that only energetic electrons will be affected by the higher order harmonics of the cyclotron resonance. Transformation to the rotating wave frame $\left(Z, P_{\|}^{\prime}, \theta^{\prime}, I^{\prime}\right)$ with the help of the generating function $F\left(z, \theta ; P_{\|}^{\prime}, I^{\prime}\right)=\theta I^{\prime}+\left[z+l \theta / k_{\|}-\pi / 2 k_{\|}-(\omega /\right.$ $\left.\left.k_{\|}\right) t\right] P_{\|}^{\prime}$ and averaging over $\theta$ brings the Hamiltonian to the canonical form

$$
\begin{gathered}
H_{0}=\left[1+2 I^{\prime} \eta+2 l P_{\|} \eta / k_{\|}+P_{\|}^{2}\right]^{1 / 2} \\
-\left(\omega / k_{\|}\right) P_{\|}^{\prime}=\gamma_{o}-\left(\omega / k_{\|}\right) P_{\|}^{\prime} \\
H_{1}\left(z, P_{\|}, I\right)=G_{l}\left(P_{\|}, I\right) \cos \left(k_{\|} Z\right)
\end{gathered}
$$$$
\text { with } \quad P_{\|}^{\prime}=P_{\|}, \quad \theta^{\prime}=\theta, \quad I=I^{\prime}+l P_{\|} / k_{\|} \quad \text { and }
$$
$Z=z+l \theta / k_{\|}-\pi / 2 k_{\|}-\left(\omega / k_{\|}\right) t$.

The new action $I^{\prime}$ is an adiabatic invariant; $\mathrm{d} I^{\prime} / \mathrm{d} t=0$. Hence, the changes in $P_{\|}$are related to changes in $I$, differently for each harmonic 1 ,

$$
\Delta I=l \Delta P_{\|} / k_{\|}
$$

The Hamiltonian equations of motion become then near the resonance

$$
\begin{aligned}
\mathrm{d} Z / \mathrm{d} t=[ & \left.\left(P_{\|}+l \eta / k_{\|}\right) / \gamma_{o}-\omega / k_{\|}\right] \\
& +\left(\partial G_{l} / \partial P_{\|}\right) \cos \left(k_{\|} Z\right) \\
\mathrm{d} P_{\|} / \mathrm{d} t= & -\left(I / \gamma_{0}\right)(\partial \eta / \partial z)+G_{l} k_{\|} \sin \left(k_{\|} Z\right)
\end{aligned}
$$

The first terms of $\mathrm{d} Z / \mathrm{d} t$ denote the "resonance" condition. When this condition is satisfied the phase $k_{\|} Z$ is approximately stationary and the electron undergoes irreversible change in $P_{\|}$and $I$. The value of the changes depends on the initial phase, leading to a 
random-phase motion in the phase space. Effective diffusion requires an inhomogeneous magnetic field, a sufficiently large wave field and energetic electrons.

\section{Simulation model}

In our model we follow test particles in the presence of an inhomogeneous magnetic field and oblique whistler waves. The dipole magnetic field is described by

$B_{r}=-2 B_{o}(R / r)^{3} \sin \lambda, \quad B_{\lambda}=B_{o}(R / r)^{3} \cos \lambda$

where $B_{o}$ denotes the surface equatorial strength, $R$ is the Earth radius and $\lambda$ denotes the latitude. The change in the guide field along the dipole coordinate $s(r, \lambda)$, where $s$ denotes the distance along the magnetic field measured from the equator, is

$$
\begin{aligned}
\mathrm{d} B / \mathrm{d} s= & B_{o} /\left(R L^{4}\right)(9 \sin \lambda)\left[1+(5 / 3) \sin ^{2} \lambda\right] \\
& \times \cos ^{-8} \lambda\left(1+3 \sin ^{2} \lambda\right)^{-1}
\end{aligned}
$$

We approximate the magnetic field by rectifying the dipole into cartesian coordinates and take the limit $\sin \lambda \ll 1$, resulting in a quadratically converging, slowly changing magnetic field

$B(x, y, z) \sim B_{e q}\left[1+z(z \hat{z}-x \hat{x}-y \hat{y}) / D^{2}\right]$

where $z$ denotes the field axis, $D \sim R L$ and $B_{e q}=B_{o} / L^{3}$ is the equatorial field. The chosen model magnetic field satisfies $\operatorname{div} B=0$ and Eq. (11) includes the effect of mirror force and implies explicitly a bounce motion along the magnetic field.

Test particle simulations and interaction with parallel propagating whistler waves for electrons with $<10 \mathrm{keV}$ were described by Chang and Inan (1985) and Inan (1987). They used coherent as well as incoherent waves and showed that the resulting precipitation fluxes are approximately similar for the two cases. Since the chorus waves have a relatively narrow spread in frequency we consider here interaction with coherent wave, which allows us to follow changes in the wave phase along the electron trajectory. We are interested in statistical properties of interaction between waves and electrons, hence we choose several pitch angles and equatorial wave propagation directions. The wave electric field is given by $\left[E_{x} \cos \psi, E_{y} \sin \psi, E_{z} \cos \psi\right]$ with $\psi=\left[\int \mathbf{k}(\epsilon z) d \mathbf{x}-\omega t+\phi\right]$, where $\phi$ is an arbitrary phase and the $\epsilon$ functional dependence emphasizes the slowly changing wave number $\mathbf{k}=(k \sin \theta, 0, k \cos \theta)$ along electron trajectory. Parallel propagation involves an electromagnetic, right-hand polarized mode, while oblique propagation makes the polarization elliptical and adds an electrostatic component. The refractive index and the wave number are recalculated each time step from the cold dispersion relation with frequency $\omega$ and propagation direction $\theta$ given. They change along electron trajectory due to the changing magnetic field, since we assume a constant density along the field line. Explicitly, the square of the refractive index $c^{2} k^{2} / \omega^{2}$ is given by $\left(B-\left(B^{2}-4 A C\right)^{0.5}\right) / 2 A$, with

$$
\begin{aligned}
& A=\epsilon_{1} \sin ^{2} \theta+\epsilon_{3} \cos ^{2} \theta \\
& B=\epsilon_{1} \epsilon_{3}+\epsilon_{1} A-\epsilon_{2}^{2} \sin ^{2} \theta \\
& C=\epsilon_{3}\left(\epsilon_{1}^{2}-\epsilon_{2}^{2}\right)
\end{aligned}
$$

where $\epsilon_{i}$ denote the standard components of the dielectric tensor (e.g. Krall and Trievelpiece, 1973).

Since energetic electrons consist of a very small density vs the subrelativistic distribution, the cold approximation is justified for the dispersion relation. Similarly, the three components of the electric field are related by the dispersion relation, such that with a prescribed $E_{x}$ all the other components are uniquely determined. The three components of the magnetic field are calculated from Faraday's law and all the resulting components of the electromagnetic field are used in the particle motion.

The simulations employ a forth-order Runge Kutta method with an adaptive time step (Press et al., 1986) and resolve the highest dynamical frequency, i.e., gyromotion. Initial wave number propagation angle is specified as well as the electron three velocities and three positions. The variables which describe the evolution of the system include particle phase space and wave phase $\psi$, which changes explicitly due to the time elapsed, the time-dependent particle position and the location-dependent wave number. Explicitly, we solve the following set of equations:

$$
\begin{aligned}
& \mathrm{d} \mathbf{x}(t) / \mathrm{d} t=\mathbf{v}(t), \\
& \mathrm{d} \mathbf{p}(t) / \mathrm{d} t=(q / m)[\mathbf{E}[x(t)]+\boldsymbol{v} \times \mathbf{B}[x(t)] / c], \\
& \mathrm{d} \psi(t) / \mathrm{d} t=k_{\|}(z) v_{\|}(t)+k_{\perp} v_{x}-\omega
\end{aligned}
$$

$\mathbf{B}(x)$ includes the guide field (Eq. 11) as well as wave fields. We integrate the relativistic electron equation of motion together with the wave phase and follow the nonadiabatic changes whenever the electron enters a resonance along its trajectory.

The propagation of the wave is not considered explicitly, since it requires a ray tracing with pathintegrated attenuation and growth of VLF waves over broad ranges of the magnetosphere. We note, however, that the existing ray tracing investigations indicate a possibility of wave trapping, their accessibility to regions far from their source generation and their large oblique propagation directions (e.g. Church and Thorne, 1983; Draganov et al., 1993). Here we take into account the time-dependent phase of a wave due to the changing gyrofrequency along electron trajectory and investigate the interaction of relativistic electrons with a whistler wave over time scale of many bounces along the magnetic field.

\section{Simulation results}

The simulations advance a system of seven variables and at given time intervals calculate several quantities which describe the irreversible changes due to the resonant interaction. In all the simulations we choose set of standard values: electric field amplitude of $1-2 \mathrm{mV} / \mathrm{m}$, 
scale length of background magnetic field $\mathrm{D}=20000 \mathrm{~km}$, equatorial magnetic field of $1.5 \times 10^{-3}$ Gauss, ratio of wave frequency to the equatorial gyrofrequency $0.2-0.4$, and we vary the initial energy, pitch angle, gyrophase and propagation direction of the whistler wave. Figure 1 describes (a) the temporal evolution of the adiabatic invariant $\mu$, (b) the kinetic energy $W=m c^{2}(\gamma-1)$ and (c) the equatorial pitch angle $\alpha$, over a short time of a fraction of a bounce period $(0.4 \mathrm{~s})$ and the few first electron resonance crossings. $\mu$, which is calculated by its lowest order approximation at the electron position $v_{\perp}^{2} / B(x)$, exhibits higher order oscillations in the adiabatic region between resonances (Catto et al., 1981), with increasing amplitude of oscillations as the electron approaches a resonance and crosses it. $\alpha$ is calculated by adiabatically projecting the instantaneous pitch angle to the equator $(z=0), \alpha=t g^{-1}\left[p_{\perp} /\left[p_{\perp}^{2}(z / D)^{2}+p_{z}^{2}\left(1+(z / D)^{2}\right)\right]^{1 / 2}\right]$. In Fig. 1d we show the calculated time-dependent mismatch to a resonance $v(t)=\left[\gamma(t) \omega-k_{\|} p_{\|}(t)\right] / \Omega(\mathbf{x}(\mathbf{t}))$. Here $\Omega[\mathbf{x}(t)] \sim q \mathbf{B}[\epsilon x(t)] / m c$ denotes the nonrelativistic gyrofrequency which changes along the particle trajectory and $\gamma$ is the relativistic factor. Between resonances the electron moves adiabatically, but when it crosses a resonance ( $v$ approaches an integer), an irreversible change in energy, in first adiabatic invariant and in equatorial pitch angle may occur. Generally the adiabatic invariant and the equatorial pitch angle undergo changes at every resonance crossing, however one observes different behaviour in $\mu$ and in $\alpha$ at the $n=0$ resonance which affects only the parallel momentum (i.e., $\alpha$ ) and energy. We note the crossing of the $n=0$ resonance at $t \sim 0.1 \mathrm{~s}$ with a large change in $\alpha$ and in $W$, without any effect on $\mu$ and the strong modification in the adiabatic invariant at higher harmonics, particularly at $n=3$ at time $t \sim 0.35 \mathrm{sec}$, which may occur only for energetic particles with gyroradii larger than the perpendicular wavelength.

Figure 2 describes the temporal evolution of the kinetic relativistic energy and the equatorial pitch angle $\alpha$, over a longer period (80 s) for three particles with different initial conditions $(W=1.5,2.5$ and $4.5 \mathrm{MeV}$;
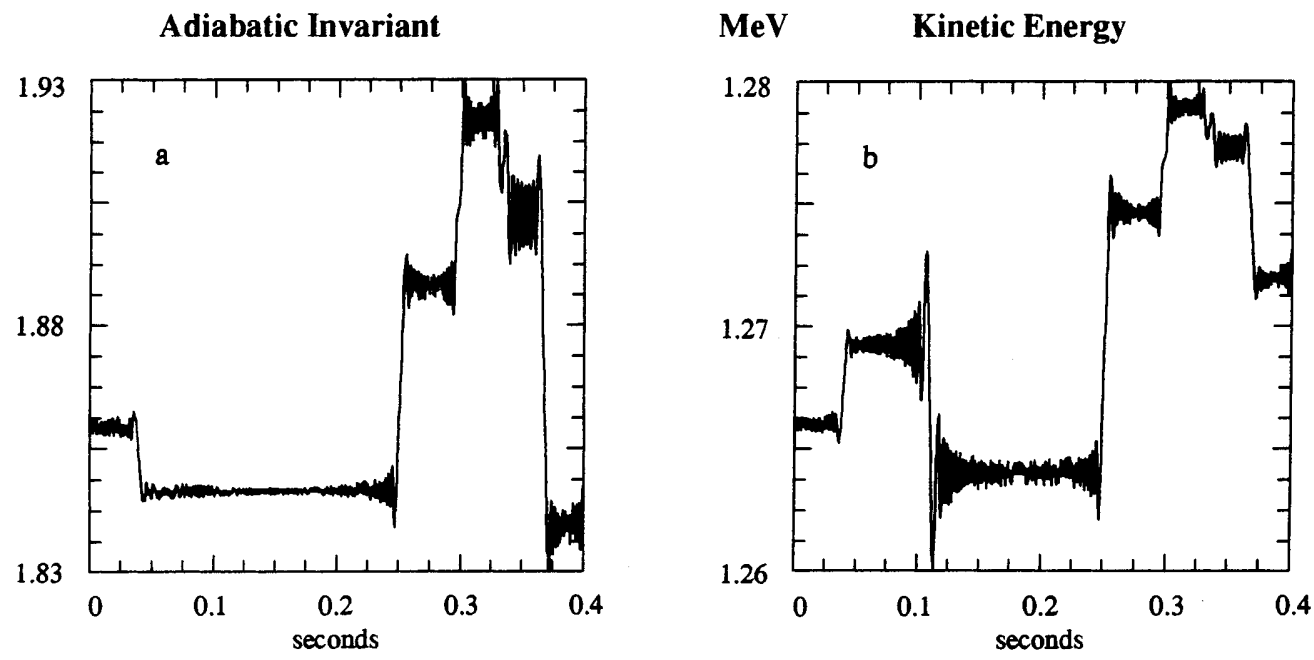

Equatorial Pitch Angle
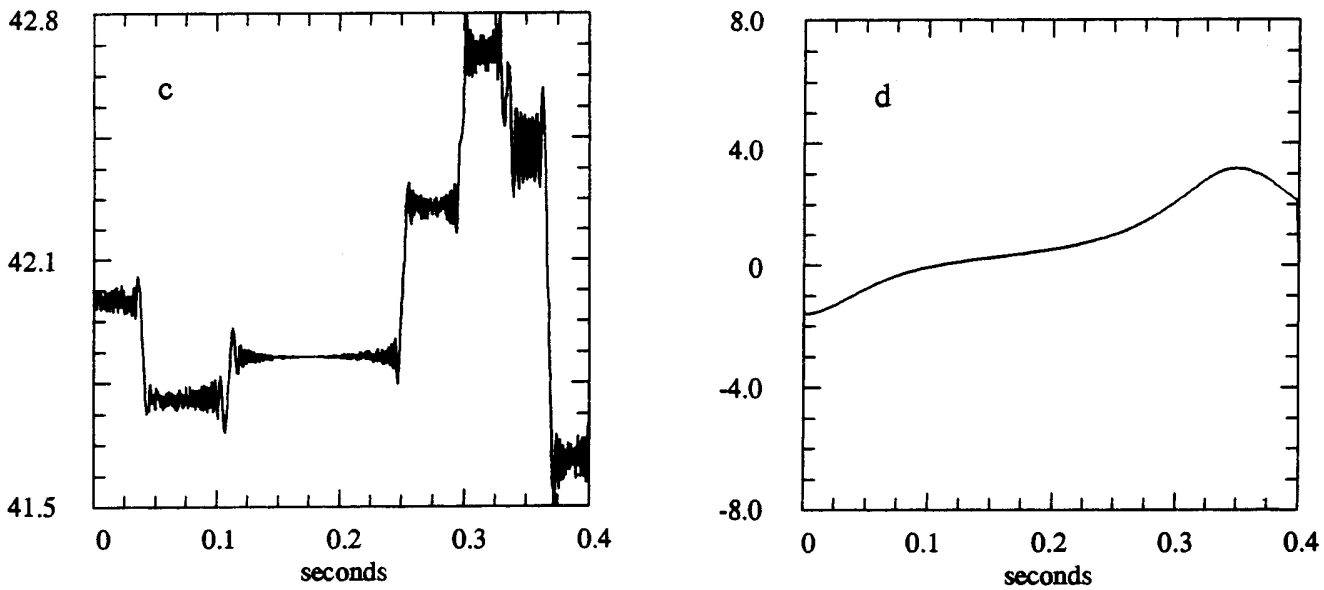

Fig. 1. (a) Adiabatic invariant, (b) kinetic energy, (c) equatorial pitch angle and (d) resonance mismatch as a function of time, for a bouncing energetic electron 
$\mathrm{MeV}$

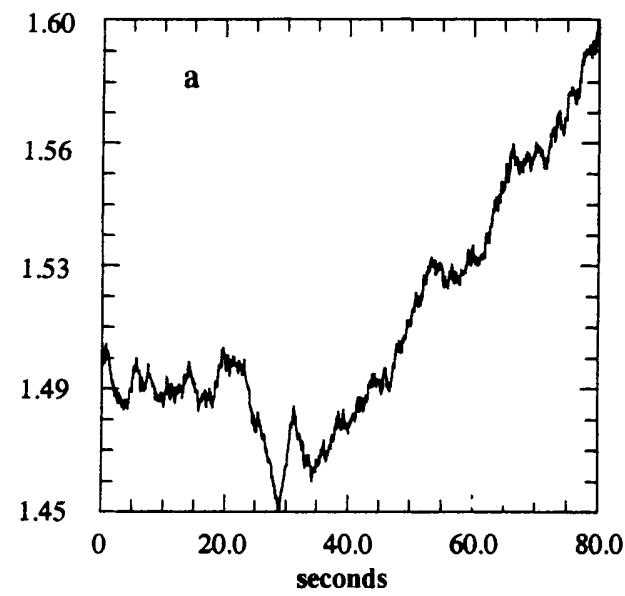

$\mathrm{MeV}$

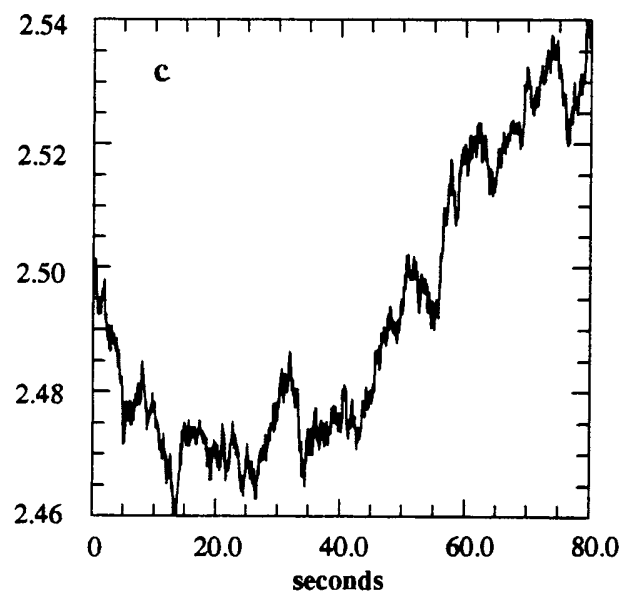

$\mathrm{MeV}$

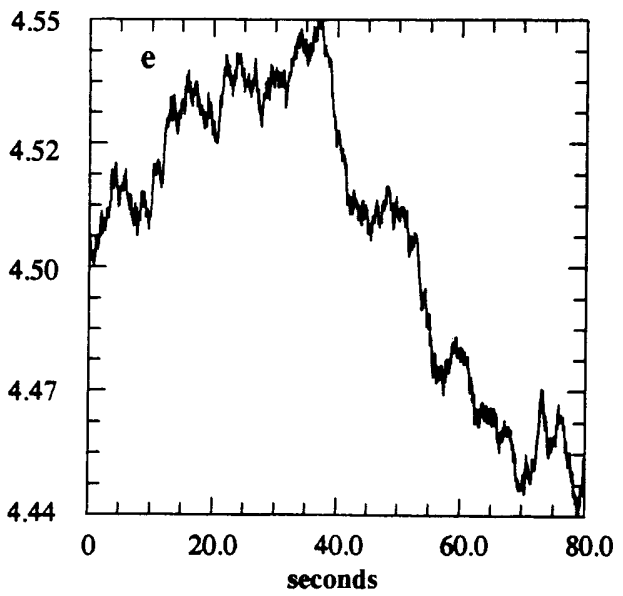

Equatorial Pitch Angle

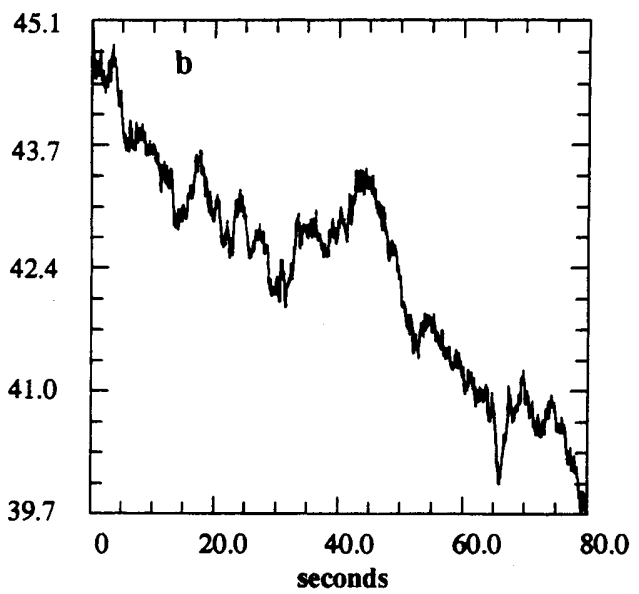

Equatorial Pitch Angle

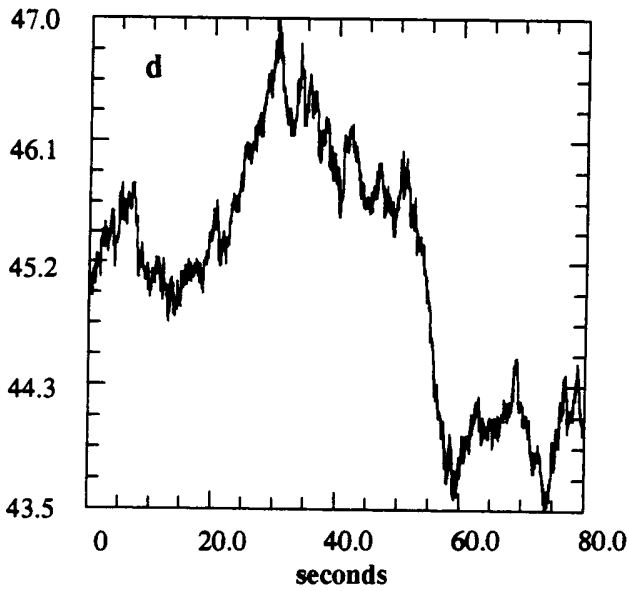

Equatorial Pitch Angle

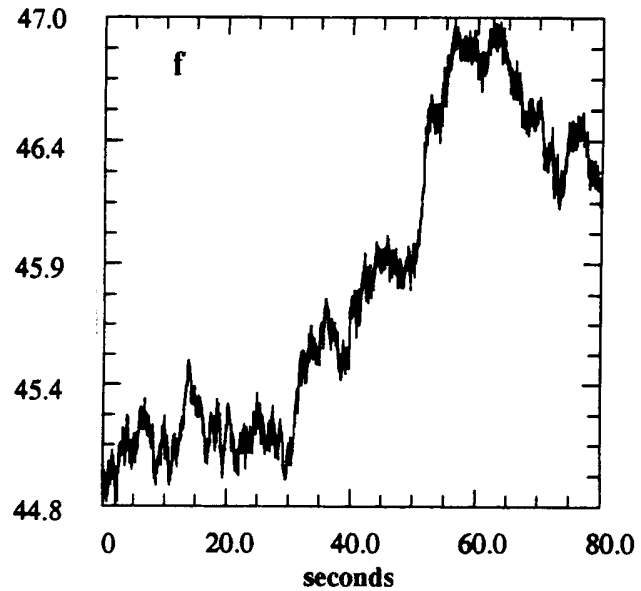

Fig. 2. Kinetic energy (W) and equatorial pitch angle $(\alpha)$ vs time for the three sample particles. $\mathrm{W}=1.5,2.5,4.5 \mathrm{MeV} ; \alpha=45^{\circ}$

$\alpha=45^{\circ}$ ). The irreversible changes in $\mu, \alpha$ and in $W$ at resonance crossings can be positive or negative and depend on the relative phase between the wave and the bouncing electron. Therefore, successive resonant interactions are a source of stochastic diffusion. One observes that during this period of time the sample electrons perform a random walk in all quantities. In the three examples shown the electron energy changes in the range $1.5-1.6,2.5-2.54$ and $4.5-4.44 \mathrm{MeV}$, respectively. At lower energies $(W<2.5 \mathrm{MeV})$ the stochastic process 
spreads the electrons in energy and pitch angles while at higher energies the dominant process is diffusion in energy. Since the seed electron distribution function generally decreases steeply in energy, the main manifestation of this diffusion may be seen as an increased flux at the higher energies.

The diffusion process depends on the amplitude of the waves and on the coupling strength at the resonances along electron trajectory, averaged over the distribution of relativistic particles. This statistical change determines a time scale for the process. Figure 3 shows the calculated square of energy change $\left(W-W_{o}\right)^{2} / \tau$ and equatorial pitch angle change $\left(\alpha-\alpha_{o}\right)^{2} / \tau$ during $\tau=8 \mathrm{~s}$ of simulation for few hundred electrons, as a function of their initial energy $W_{o}$. The initial conditions cover three pitch angles $\alpha_{o}\left(30^{\circ}, 45^{\circ}\right.$ and $\left.60^{\circ}\right)$ and energies $W_{o}$ in increments of $50 \mathrm{keV}$ in the range $0.5-8.0 \mathrm{MeV}$. The chosen wave amplitude is $2 \mathrm{mV} / \mathrm{m}$ with an equatorial wave number inclined by $30^{\circ}$ with respect to the magnetic field. One observes a statistically homogeneous energy diffusion with a small increase as a function of the initial electron energy, while the diffusion in pitch angle decreases with the initial energy, indicating a lack of correlation between the two quantities. For a field of $2 \mathrm{mV} / \mathrm{m}$ and power-law index of 3-5 the time scale for an enhancement of relativistic electrons becomes 30 $60 \mathrm{~min}$. This time is much smaller than any $L$-shell diffusion time scale. Since the electron diffusion increases quadratically with the electric field amplitude, during strong geomagnetic events the diffusion of relativistic electrons due to interaction with whistlers may occur over time scales of minutes. Therefore the pulses of whistler VLF waves, as observed in conjunction with the substorm related injections, can partially explain the increase in the relativistic fluxes of outer zone electrons.

\section{Discussion and summary}

The phase space of a near-integrable dynamical system with two degrees of freedom consists of regular regions and chaotic domains which surround the separatrices around resonances. With the increase in the perturbation amplitude the chaotic regions cover an increasingly larger phase space and in a presence of a large amplitude wave overlapping between resonance layers leads to a global stochasticity (e.g., Lichtenberg and Lieberman, 1992). Time-independent Hamiltonian with two degrees of freedom is dynamically equivalent to a time-dependent Hamiltonian with one degree of freedom, which applies to an electron in a homogeneous magnetic field interacting with a wave field. Since crossing a resonance can be described with the help of a standard nonlinear pendulum, the stochastic motion in a homogeneous magnetic field requires large oscillations around the resonance, with a need of a very intense wave and a possibility of tunneling to an adjacent resonance. In the radiation belt the additional degree of freedom due to the bounce along the inhomogeneous magnetic field allows the relativistic electron to move adiabatically between consecutive resonances and interact at resonances, without a need of strong nonlinearity. For the energetic electrons the couplings at all resonances are of the same order of magnitude. Therefore much smaller amplitude wave is able to diffuse the electrons in pitch angle and in energy. The bouncing along the inhomogeneous magnetic field and the obliqueness of the waves are the requirements for an effective stochastic diffusion in the first two adiabatic invariants and in energy.

The enhancement of relativistic electrons at geomagnetically active periods (as the recovery stage of the geomagnetic storm) may be due to a resonant interaction with the whistler chorus waves. These waves, which can be ducted to the observing instrument on ground, exist in the quiet magnetosphere as continually repeated narrow-band signals, and are enhanced during active substorm periods in a typical rising-frequency mode. They constitute a characteristic feature of substorms and are generated by the $10-100 \mathrm{keV}$ electrons which are injected during its expansion phase. The seed of energetic, relativistic electrons interacts resonantly with these waves along their bounce trajectories in the
$(\mathrm{MeV})^{2} / \mathrm{s} \quad$ Energy Diffusion

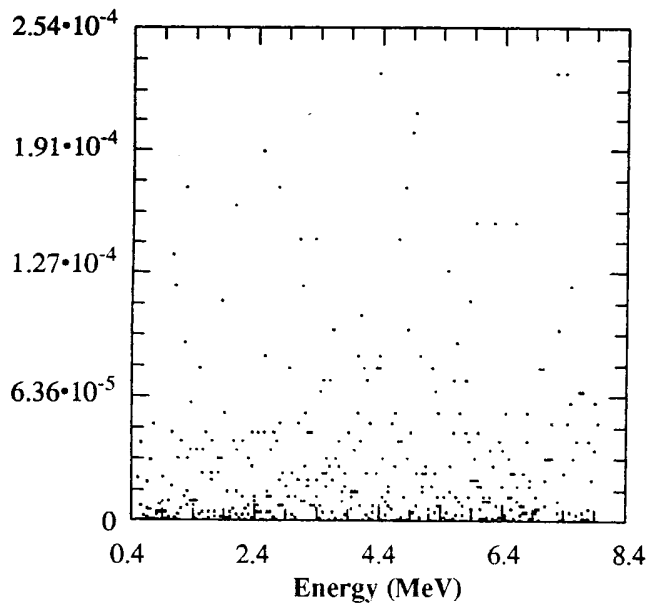

Pitch Angle Diffusion

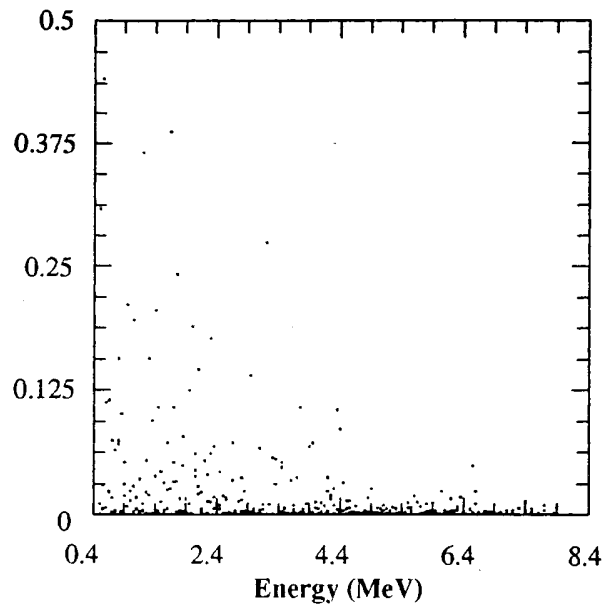

Fig. 3. Diffusion in energy and in pitch angle for several hundreds of electrons as a function of the initial energy 
inhomogeneous magnetic field. An interaction around a given resonance follows an adiabatic motion to the adjacent resonance and the couplings at all the harmonics are of the same order of magnitude. The interaction with higher harmonics is effective only for relativistic electrons and the time scale of the resulting stochastic diffusion is much faster than radial diffusion. Therefore this process of enhancement of relativistic electrons couples substorm injections events with intense geomagnetic perturbations.

Acknowledgements. We acknowledge the support of the NASA grants NAG5-3182, NAG5-2815 and NAG5-3596.

Topical Editor K.-H. Glassmeier thank P. Gary for his help in evaluating this paper.

\section{Reference}

Baker D. N. et al., Recurrent Geomagnetic storms and relativistic electrons: enhancements in outer magnetosphere: ISTP coordinated measurements, J. Geophys. Res., 102, 14141, 1997.

Blake, J. B. et al., CEPPAD: Comprehensive energetic particle and pitch angle distribution experiment on POLAR, Space Sci. Rev., 71, 531, 1996.

Catto, P. J., W. M. Tang, and D. E. Baldwin, Generalized gyrokinetics, Plasma Phys, 23, 639, 1981.

Chang, H. C., and U. S. Inan, Test particle modeling of waveinduced energetic electron precipitation, J. Geophys. Res., 90, 6409, 1985

Church, S. R., and R. M. Thorne, On the origin of plasmaspheric hiss: ray path integrated amplification, J. Geophys. Res., 88, 7941, 1983.

Draganov, A. B., U. S. Inan, V. S. Sonwalker, and T. F. Bell, Whistlers and plasmaspheric hiss: wave directions and threedimensional propagation, J. Geophys. Res., 98, 11401, 1993.

Hasegawa, A., Plasma instabilities and nonlinear effects, SpringerVerlag, Berlin Heidelberg New York, 1975.

Hudson, M. K., S. R. Elkington, J. G. Lyon, C. C. Goodrich, and T. J. Rosenberg, Simulation of radiation belt dynamics driven by solar wind variation, in "The Physics of Sun-Earth Plasma" J. L. Burch, R. L. Carorillano and S. Antiochos, eds AGU, in press, 1999

Jasna, D., U. S. Inan, and T. F. Bell, Precipitation of suprathermal $(100 \mathrm{eV})$ electrons by oblique whistler waves, Geophys. Res. Lett., 19, 1639, 1992.

Inan, U. S., Gyroresonant pitch angle scattering by coherent and incoherent whistler mode waves in the magnetosphere, J. Geophys. Res., 92, 127, 1987.

Li, X., I. Roth, M. Temerin, J. R. Wygant, M. K. Hudson, and J. B. Blake, Simulation of the prompt energization and transport of radiation belt particles during the March 24, 1991 SSC, Geophys. Res. Lett., 20, 2423, 1993.

Li, X., D. N. Baker, M. Temerin, J. R. Wygant, M. K. Hudson, and J. B. Blake, Are energetic electrons in the solar wind the source of the outer radiation belt? Geophys. Res. Lett., 24, 8, 923, 1997.

Li, X., D. N. Baker, M. Temerin, J. R. Wygant, M. K. Hudson, and J. B. Blake, Satellite observations of the outer zone electron variation during the 3-4 November 1993 magnetic storm, Geophys. Res. Lett., in press, 1998.

Lichtenberg, A. J. and M. A. Lieberman, Regular and chaotic dynamics, New York: Springer-Verlag, 1992.

Lyons, L. R. and R. M. Thorne, Equilibrium structure of radiation belt electrons, J. Geophys.Res., 78, 2142, 1973.

Krall, N. A., and A. W. Trivelpiece, Principles of plasma physics, McGraw Hill, 1973.

McIlwain, C. E., Processes action upon outer szone electrons in Radiation belts: models and standards, Ed. J. F. Lemaire, D Heynderickx and D. N. Baker, pp. 15-26, AGU, 1996.

Paulikas, G. A. and J. B. Blake, Effects of the solar wind on magnetospheric dynamics: Energetic electrons at the synchronous orbit, in Quantitative Modeling of Magnetospheric Processes, 21, Geophys. Monograph Series, 1979.

Press, W. H., B. P. Flannery, S. A. Teukolsky, and W. T. Vetterling, Numerical Recipes, Cambridge University Press, Cambridge, 1986.

Reeves, G. D., R. H. W. Friedel, M. G. Henderson, R. D. Belian, M. M. Meier, D. N. Baker, T. Onsager, and H. J. Singer, The relativistic electron response at geosynchronous orbit during the January 1997 magnetic storm, J. Geophys. Res., 103, 17559, 1998.

Ristic-Djurovic, J. L., T. F. Bell and U. S. Inan, Precipitation of radiation belts electrons by magetospherically reflected whistler, J. Geophys. Res., 103, 9249, 1998.

Roederer, J. G., Dynamics of Geomagnetically Trapped Radiation, Springer-Verlag, New York Berlin Heidelberg, 1970.

Schulz, M., and L. J. Lanzerotti, Particle diffusion in the radiation belts, 215 pp., Springer-Verlag, New York Berlin Heidelberg 1974.

Schulz, M., The magnetosphere, in Geomagnetism, vol 4, Ed. J. A. Jacobs, pp. 87-293, Academic Press, London, 1991.

Selesnick, R. S., and J. B. Blake, Dynamics of the outer radiation belts, Geophys. Res. Lett., 24, 1347, 1997.

Selesnick, R. S., and J. B. Blake, Radiation belt observations from January 6 to 20, 1997, Geophys. Res. Lett., in press, 1998.

Selesnick, R. S., J. B. Blake, and W. A. Kolasinski, A quiescent state of 3 to $8 \mathrm{MeV}$ radiation belt electrons, Geophys. Res. Lett., 24, 1343, 1997.

Smith, A. J., M. P. Freeman, and G. D. Reeves, Postmidnight VLF chorus events, a substorm signature observed at the ground near $L=4, J$. Geophys. Res., 101, 24641, 1996.

Thorne, R. M., and R. B. Horne, Landau damping of magnetospherically reflected whistlers, J. Geophys. Res., 99, 17249, 1994.

Thorne, R. M., and R. B. Horne, Whistler absorption and electron heating near the plasmapause, J. Geophys. Res., 101, 4917, 1996.

Tsurutani, B. T., and E. J. Smith, Two types of magnetospheric chorus and their substorm dependences, J. Geophys. Res., 82, $5112,1977$. 